\title{
Focos de Enfermagem em pessoas mais velhas com problemas de saúde mental
}

\author{
Nursing focuses in older people with mental disorders \\ Focos de Enfermería en las personas mayores con problemas de salud mental \\ Joaquim Passos* \\ Carlos Sequeira** \\ Lia Fernandes***
}

\section{Resumo}

Enquadramento: As perturbações mentais nos idosos associam-se frequentemente a perdas cognitivas, sintomas psicológicos e comportamentais que comprometem a sua qualidade de vida, constituindo manifestações relevantes para a prática de Enfermagem. Objetivo: Identificar os focos de Enfermagem relacionados com a saúde mental mais comuns nas pessoas mais velhas.

Metodologia: Foram avaliados 75 utentes ( $\geq 65$ anos), com doença mental diagnosticada pela Classificação Internacional de Doenças, $9^{a}$ Revisão (CID-9), num Departamento de Psiquiatria e Saúde Mental. Instrumentos: Mini-Exame do Estado Mental; Teste do Relógio; Escala de Depressão Geriátrica; Escala de Ansiedade de Zung; Indice de Barthel; Índice de Lawton e Brody; e Classificação Social de Graffar.

Resultados: Diagnósticos médicos mais comuns: depressão e demência. A maioria dos utentes apresentava défice cognitivo, depressão, ansiedade, dependência moderada nas Atividades Básicas de Vida Diária e algum nível de dependência nas Atividades Instrumentais de Vida Diária. Focos de Enfermagem mais comuns: memória, cansaço, ansiedade, nervosismo, insónia e atenção. Conclusão: As alterações cognitivas, a demência e a depressão estavam associadas a maior número de focos de Enfermagem.

Palavras-chave: enfermagem psiquiátrica; foco de enfermagem; diagnóstico de enfermagem; saúde mental; idoso; idoso de 80 anos ou mais.

\begin{abstract}
Background: Mental disorders in the elderly are often associated with cognitive losses, and psychological and behavioural symptoms which compromise their quality of life. These manifestations are relevant to Nursing practice.

Objective: To identify the most common mental health-related Nursing focuses among older people.

Methodology: A sample of 75 users ( $\geq 65$ years old), with mental illness diagnosed using the International Classification of Diseases, Ninth Revision (ICD-9), in a Psychiatry and Mental Health Unit was assessed. The instruments used were: the Mini-Mental State Examination; the Clock-Draw Test; the Geriatric Depression Scale; the Zung Anxiety Scale; the Barthel Index; the Lawton and Brody Index; and the Graffar's Social Classification. Results: The most common medical diagnoses were depression and dementia. Most users had cognitive impairments, depression, and anxiety, and showed moderate dependence in Basic Activities of Daily Living and some level of dependence in Instrumental Activities of Daily Living. The most common Nursing focuses were memory, fatigue, anxiety, nervousness, insomnia and attention.

Conclusion: Cognitive changes, dementia and depression were associated with a higher number of Nursing focuses.
\end{abstract}

Keywords: psychiatric nursing; nursing focus; nursing diagnosis; mental health; aged; aged, 80 and over.

\footnotetext{
* Enfermeiro Especialista em Enfermagem de Saúde Mental e Psiquiatria. Doutorando em Gerontologia pelo ICBAS-UP/UA. Professor Assistente Convidado da Escola Superior de Saúde do Instituto Politécnico de Viana do Castelo, Escola Superior de Saúde, 4900-314, Viana do Castelo,
Portugal [ joaquimpassos@ hotmail.com]. Morada para correspondência: Escola Superior de SaúdeRua D. Moisés Alves de Pinho, 4900-314, Viana do Castelo, Portugal

** Professor Coordenador da Escola Superior de Enfermagem do Porto, 4200-072, Porto, Portugal. Coordenador da Unidade Cientifico-Pedagógica: "Gestão de Sinais e Sintomas". Coordenador do Grupo de Investigação "NurID: Inovacão e Desenvolvimento em Enfermagem" - CINTESISdo Grupo de Investigação "NurID: Inovação e Desenvolvimento em Enfermagem" - CINTESIS-
FMUP. Diretor da Revista Portuguesa de Enfermagem de Saúde Mental. Presidente da Sociedade FMUP. Diretor da Revista Portuguesa de Enfermagem de Saúde Mental.

* * Professora Associada da Faculdade de Medicina da Universidade do Porto / Psiquiatra do Centro Hospitalar de São João do Porto. Doutora em Medicina, Faculdade de Medicina da Universidade do Porto, 4200 -319 Porto, Portugal [lfernandes@med.up.pt].
}

\section{Resumen}

Marco contextual: Los trastornos mentales en las personas mayores se asocian con problemas cognitivos, síntomas psicológicos y de comportamiento que afectan a la calidad de vida de estas personas y son relevantes para la práctica de enfermería.

Objetivo: Identificar los focos de Enfermería relacionados con la salud mental más comunes en las personas mayores.

Metodología: Fueron evaluados 75 usuarios ( $\geq 65$ años), con diagnóstico de enfermedad mental por la Clasificación Internacional de Enfermedades, 9 revisión (CIE-9), en un Departamento de Psiquiatría y Salud Mental. Instrumentos: MiniExamen del Estado Mental; Test del Reloj; Escala de Depresión Geriátrica; Escala de Ansiedad de Zung; Índice de Barthel; Índice de Lawton y Brody; y Escala de Clasificación Social de Graffar. Resultados: Los diagnósticos médicos más comunes son depresión y demencia. La mayoría de los usuarios presentaba deterioro cognitivo, depresión, ansiedad, dependencia moderada en las Actividades Básicas de la Vida Diaria y un cierto grado de dependencia en las Actividades Instrumentales de la Vida Diaria. Los focos de Enfermería más comunes son memoria, fatiga, ansiedad, nerviosismo, insomnio y atención.

Conclusión: Los trastornos cognitivos, la demencia y la depresión se asociaron con un mayor número de focos de Enfermería.

Palabras clave: enfermería psiquiátrica; foco de enfermería; diagnóstico de enfermería; salud mental; anciano; anciano de 80 o más años.

Recebido para publicação em: 13.01 .14

Aceite para publicação em: 04.03 .14 


\section{Introdução}

A tendência de envelhecimento da população tem vindo a acentuar-se em Portugal, à semelhança da maioria dos países europeus, aumentando significativamente o número de pessoas mais velhas na população portuguesa. Esta tendência reforça a problemática relacionada com o envelhecimento e coloca maiores preocupações em relação às condições de vida e de saúde/doença das pessoas mais velhas.

Associada a estas evidências, a prevalência de doenças crónicas, com a coexistência de comorbilidade física e mental, nesta população, constitui um fator que contribui para a agudização dos seus problemas. No que diz respeito à doença mental, os dados disponíveis apontam para um incremento das perturbações depressivas e dos quadros demenciais, traduzidos por manifestações psicopatológicas, muitas vezes apresentadas sob a forma de síndromes clínicos, que dificultam o processo de avaliação (Canabrava et al., 2012). Estes dados suscitam uma análise aprofundada, de modo a conhecer a sua verdadeira natureza e a traduzi-los em constructos sensíveis à terminologia de Enfermagem. Muitas destas condições constituem domínios ou focos relevantes para a prática clínica (Passos, Sequeira, \& Fernandes, 2010, 2012; Sequeira, 2010), devendo ser reconhecidos pelos enfermeiros, tendo por base uma linguagem comum, que permita sustentar a padronização da documentação e a consequente valorização da prática, através da identificação de diagnósticos, intervenções e resultados sensíveis aos cuidados de Enfermagem (Conselho Internacional de Enfermeiros, 2011). No sentido de responder a algumas destas preocupações, foi delineado o presente estudo, que teve como principal objetivo identificar focos de atenção de Enfermagem, relacionados com a saúde mental, mais comuns nas pessoas mais velhas com doença mental. Pretendia-se também analisar a relação entre estes focos e os fatores sociodemográficos, clínicos e de funcionalidade. Procurou-se ainda dar um contributo para a elaboração de um Catálogo CIPE (Classificação Internacional para a Prática de Enfermagem) em saúde mental, dirigido às necessidades do idoso, e para o desenvolvimento de um questionário de avaliação clínica de Enfermagem de saúde mental e psiquiatria. Para o enquadramento deste trabalho utilizou-se a CIPE, uma vez que é a classificação proposta pelo Conselho Internacional de Enfermeiros
(CIE) e pela Ordem dos Enfermeiros Portugueses e faz parte das classificações internacionais reconhecidas pela Organização Mundial de Saúde (OMS), sendo largamente utilizada nos sistemas de informação de apoio à prática clínica, em Portugal e a nível internacional (Conselho Internacional de Enfermeiros, 2011).

\section{Enquadramento}

De acordo com Benedetti, Borges, Petroski, e Gonçalves (2008), as perturbações mentais comprometem $20 \%$ da população mais velha, entre as quais se destacam a demência e a depressão com maior prevalência. O envelhecimento populacional tem levado a um aumento na prevalência de doenças degenerativas crónicas, especialmente da demência, assumindo a manutenção da saúde cognitiva uma importância relevante para a prevenção da dependência e da incapacidade funcional (Apóstolo, Cardoso, Marta, \& Amaral, 2011), originando também um incremento da doença física e dos problemas sociais. Do mesmo modo, a depressão é descrita como uma doença incapacitante, com consequências a nível do funcionamento global e da satisfação das necessidades da pessoa mais velha (Benedetti et al., 2008). Associadas a estas condições psicopatológicas, ocorre um conjunto de perdas cognitivas, sobretudo nas pessoas de idade mais avançada, bem como outros sintomas psicológicos e comportamentais que determinam problemas e necessidades específicas.

Neste sentido, os enfermeiros, devem estudar e analisar esta problemática numa perspetiva holística e multidimensional, de modo a identificar os focos mais relevantes para a Enfermagem e a criar evidência científica que permita melhorar a resposta ao nível da prevenção, tratamento e reabilitação das pessoas mais velhas com problemas de saúde mental. Neste domínio, o CIE sugere o envolvimento dos enfermeiros na investigação e realça a necessidade da existência de dados, baseados na evidência, que permitam criar padrões para a representação da prática em Enfermagem nos sistemas de informação em saúde baseada no conhecimento e no contexto dos cuidados de saúde globais (Conselho Internacional de Enfermeiros, 2011). Em consequência, incentiva os enfermeiros, nos diferentes contextos da sua intervenção, a identificarem focos relevantes para a 
prática de Enfermagem, relacionados com prioridades de saúde ou grupos e populações específicas, de modo a contribuírem para a criação de catálogos CIPE e para a melhoria desta classificação (Conselho Internacional de Enfermeiros, 2011). Os focos constituem áreas de atenção relevantes para a Enfermagem (por exemplo, ansiedade, confusão, tristeza, solidão, autoestima, etc.) e são fundamentais para que o enfermeiro estabeleça o diagnóstico de Enfermagem, que constitui a base para o planeamento da assistência (Conselho Internacional de Enfermeiros, 2011). A identificação correta de um foco de Enfermagem é um passo essencial para o reconhecimento de um problema ou de uma necessidade específica e para a elaboração de um diagnóstico, que permita planear uma resposta adequada.

\section{Questóes de Investigação}

Quais os focos de Enfermagem relacionados com a saúde mental mais comuns nas pessoas mais velhas com doença mental?

Qual a relação entre estes focos e os fatores sociodemográficos, clínicos e de funcionalidade?

\section{Metodologia}

Tendo em conta o propósito da investigação e os objetivos enunciados, foi realizado um estudo descritivo e uma análise exploratória, utilizando uma abordagem quantitativa e correlacional.

\section{Participantes}

Foi recrutada uma amostra de 75 participantes, com idade igual ou superior a 65 anos, de ambos os sexos, com doença mental diagnosticada segundo a Classificação Internacional de Doenças, 9a Revisão (CID-9) (World Health Organization, 1978) no contexto do Departamento de Psiquiatria e Saúde Mental (DPSM), internamento e consulta externa, da Unidade Local de Saúde do Alto Minho, EPE (ULSAM, EPE).

Foram excluídos os utentes que apresentavam défice visual ou auditivo, bem como grave compromisso na comunicação, que impedissem a aplicação do protocolo de avaliação.

\section{Instrumentos}

De modo a adequar o protocolo de avaliação ao contexto e ao propósito da investigação, foram selecionados instrumentos ajustados ao objetivo do estudo e às caraterísticas da população e utilizadas versões validadas ou adaptadas para a população portuguesa. Foram usados os seguintes instrumentos: 1) Mini-Exame do Estado Mental (Mini-Mental State Examination -MMSE), considerando os novos pontos de corte adaptados para a população portuguesa (Morgado, Rocha, Maruta, Guerreiro, \& Martins, 2009), sendo que a Defeito cognitivo correspondem os scores de: 0 - 2 anos de escolaridade $\leq 22 ; 3$ - 6 anos de escolaridade $\leq 24 ; 7$ ou mais anos de escolaridade $\leq 27 ; 2)$ Teste do Relógio (O Clock Draw Test - CDT), tendo como pontos de corte de $0-10:>6$ - normal e $\leq 6$ - anormal (Cacho, García-García, Arcaya, Vicente, \& Lantada,1999); 3) Escala de Depressão Geriátrica (Geriatric Depression Scale - GDS - 15 itens), com scores de sem depressão $\leq 5$ e com depressão $>5$ (Barreto, Leuschner, Santos, \& Sobral, 2008); 4) Escala de Ansiedade de Zung (Zung Anxiety Scale - ZAS), seguindo o ponto de corte de sem ansiedade $<40$ e com ansiedade $\geq 40$ (Serra, Ponciano, \& Relvas, 1982); 5) Índice de Barthel (Barthel Index - BI), cujos pontos de corte correspondem a: Independência total com 20; Dependência moderada de 13 - 19; Dependência grave de 9 - 12; e Total dependência de 0 - 8 (Araújo, Ribeiro, Oliveira, \& Pinto, 2007); 6) Índice de Lawton e Brody (Lawton \& Brody Index - LI), com os valores de Independência total - 23 e Algum nível de dependência < 23 (Araújo, Ribeiro, Oliveira, Pinto, \& Martins, 2008); e 7) Classificação Social de Graffar (Graffar's Social Classification GSC), considerando a seguinte divisão: 0 - 2 classe I; 3 - 4 classe II; 5 - 6 classe III; 7 - 8 classe IV; e 9 - 10 classe V (Fernandes, 2004).

\section{Procedimento}

Os participantes foram identificados através da listagem de consultas programadas. As entrevistas de avaliação foram realizadas pelo investigador, no serviço de internamento ou nas unidades de consulta externa, no período de abril a setembro de 2011. O investigador forneceu informação acerca do estudo, convidando os utentes à participação com consentimento informado. Foram avaliados 21 utentes internados, 46 na consulta externa, 6 em lares e 2 no domicílio. Alguns utentes foram avaliados em lares ou no seu domicílio devido 
a problemas físicos ou de saúde que impediam a sua deslocação aos serviços. Foram ainda entrevistados 52 cuidadores e colhida informação dos técnicos de saúde de 71 doentes (psiquiatras, psicólogos, assistentes sociais e enfermeiros).

Para a caraterização sociodemográfica e clínica recorreu-se aos registos da informação em formulário próprio, que fez parte do protocolo de investigação. Para completar esta caraterização, além das entrevistas de avaliação realizadas, foi também efetuada uma consulta ao Sistema de Apoio à Prática de Enfermagem (SAPE) e ao Sistema de Apoio ao Médico (SAM), bem como ao processo individual de cada utente.

Relativamente à informação clínica, foram ainda realizadas entrevistas informais aos psiquiatras, no sentido de atualizar a informação acerca do diagnóstico médico, e aos enfermeiros, para analisar a informação relativa aos focos de Enfermagem relacionados com a saúde mental e aos critérios de seleção e de parametrização utilizados.

No que diz respeito à informação clínica de Enfermagem, foram considerados apenas os focos de atenção relacionados com a saúde mental, tendo estes sido classificados de acordo com a CIPE, versão 2 (Conselho Internacional de Enfermeiros, 2011).

Tendo por base a informação obtida a partir da aplicação do protocolo de avaliação, bem como dos outros procedimentos descritos anteriormente, procurou-se identificar os focos de atenção relevantes para a prática de Enfermagem relacionados com a saúde mental mais comuns na população em estudo. Neste sentido, foram consideradas as pontuações globais e parciais dos instrumentos utilizados, bem como a informação obtida a partir da análise de itens, ou grupos de itens, e de diferentes domínios dos instrumentos que, de forma direta ou conjugada, permitissem identificar áreas ou focos clínicos relacionados com a saúde mental e com interesse para a prática de Enfermagem. Utilizando a CIPE (versão 2), procurou-se valorizar a informação obtida, tendo em conta a descrição do conceito de cada foco de Enfermagem relacionado com a saúde mental para, desse modo, selecionar os que apresentavam alterações ou algum nível de compromisso.

Foi obtida autorização da Comissão de Ética e da Direção da Instituição onde decorreu o estudo, bem como o consentimento informado dos utentes e cuidadores informais e acauteladas as medidas necessárias para salvaguarda da confidencialidade.

\section{Análise estatística}

Os dados foram organizados e classificados, procedendo-se ao seu tratamento estatístico utilizando o software Statistical Package for the Social Sciences (SPSS), versão 19,0. Para analisar a relação entre as principais variáveis foram utilizados testes de Quiquadrado $\left(X^{2}\right)$ e o Coeficiente de Correlação Ponto Bisserial $\left(r_{p b}\right)$. Foi ainda utilizado o Coeficiente de Correlação Parcial para analisar algumas correlações, controlando-as relativamente a outros fatores. Foi considerado um nível de significância de 0,05.

\section{Resultados}

Os dados foram obtidos a partir de uma amostra de 75 utentes, com idades entre 65 e 93 anos $(M=73,3$ anos; DP $=6,6)$ e escolaridade entre 0 e 9 anos $(M$ $=2,76$ anos; $\mathrm{DP}=1,96)$. Na amostra, 73,3\% eram do género feminino, casadas (49,3\%), viviam com o companheiro $(50,7 \%)$, em áreas rurais $(74,7 \%)$ e pertenciam a classe social muito baixa $(94,7 \%)$. Foram avaliadas no contexto da consulta externa (73,3\%), tinham como diagnóstico principal perturbação depressiva (36,0\%) e demência (29,3\%), e apresentavam comorbilidade somática em 98,7\% dos casos. Grande parte dos participantes $(69,3 \%)$ tinha cuidador informal e vivia com o cuidador $(56,0 \%)$.

\section{Resultados sobre a condição cognitiva, psicológica e de funcionalidade}

A pontuação global dos instrumentos utilizados revelou que 52,0\% dos participantes apresentavam défice cognitivo na avaliação com o MMSE $(M=22,28$; $\mathrm{DP}=6,45)$ e $66,7 \%$ tinham um resultado anormal no CDT $(M=4,65 ; \mathrm{DP}=3,40)$. Apresentavam também depressão (GDS - 61,3\%), pontuação média de 8,14 $(\mathrm{DP}=4,32)$, e 81,3\% apresentavam ansiedade na ZAS $(M=48,49 ; \mathrm{DP}=8,41)$. No que diz respeito à funcionalidade, 49,3\% apresentavam dependência moderada nas Atividades Básicas de Vida Diária (ABVD) avaliadas pelo BI, 42,7\% independência total e 4,0\% apresentava dependência grave ou total dependência $(M=17,52 ; \mathrm{DP}=3,68)$. Relativamente às Atividades Instrumentais de Vida Diária (AIVD) avaliadas pelo LI, 77,3\% apresentavam algum nível de dependência $(M=13,41 ; \mathrm{DP}=8,05)$. 
Focos de Enfermagem relacionados com a área de saúde mental

Os resultados permitiram identificar 42 focos de atenção em Enfermagem relacionados com a saúde mental, ao nível dos quais se encontraram alterações e que apresentavam maior probabilidade de se constituírem como problema de saúde nas pessoas mais velhas com doença mental. Através da análise efetuada foi possível reconhecer diferentes condições clínicas ao nível dos focos identificados (atual, anormal, comprometido, deteriorado, diminuído, elevado, etc.), o que permitiu considerá-los como áreas de atenção relevantes nesta população. A Tabela 1 apresenta a frequência dos focos mais comuns, em relação à amostra total e aos grupos de ambulatório e internamento. Foram identificados como presentes os focos de Enfermagem ao nível dos quais a pessoa apresentava alguma alteração, sendo nos restantes casos considerados como ausentes. No que diz respeito à amostra total, os focos mais frequentes foram memória (90,7\%), cansaço (86,7\%), ansiedade (84,0\%), nervosismo $(78,7 \%)$ e insónia (77,3\%). Os doentes avaliados em ambulatório apresentavam como focos mais comuns memória $(92,7 \%)$, cansaço (87,3\%), ansiedade (83,6\%), insónia e nervosismo (78,2\% cada), atenção (67,3\%) e solidão (63,6\%). Nos utentes avaliados no internamento, os focos mais frequentes foram atenção $(95,0 \%)$, pensamento (90,0\%), ansiedade, memória, concentração, cansaço e cognição ( $85,0 \%$ cada).

Tabela 1

Frequência dos focos de Enfermagem

\begin{tabular}{|c|c|c|c|c|c|c|c|c|c|c|c|c|}
\hline \multirow{3}{*}{$\begin{array}{l}\text { Focos de } \\
\text { Enfermagem }\end{array}$} & \multicolumn{4}{|c|}{ Amostra total $(n=75)$} & \multicolumn{4}{|c|}{ Ambulatório $(n=55)$} & \multicolumn{4}{|c|}{ Internamento $(n=20)$} \\
\hline & \multicolumn{2}{|c|}{ Presente } & \multicolumn{2}{|c|}{ Ausente } & \multicolumn{2}{|c|}{ Presente } & \multicolumn{2}{|c|}{ Ausente } & \multicolumn{2}{|c|}{ Presente } & \multicolumn{2}{|c|}{ Ausente } \\
\hline & $\mathrm{n}$ & $\%$ & $\mathrm{n}$ & $\%$ & $\mathrm{n}$ & $\%$ & $\mathrm{n}$ & $\%$ & $\mathrm{n}$ & $\%$ & $\mathrm{n}$ & $\%$ \\
\hline Ansiedade & 63 & 84,0 & 12 & 16,0 & 46 & 83,6 & 9 & 16,4 & 17 & 85,0 & 3 & 15,0 \\
\hline Atenção & 56 & 74,7 & 19 & 25,3 & 37 & 67,3 & 18 & 32,7 & 19 & 95,0 & 1 & 5,0 \\
\hline Auto-estima & 31 & 41,3 & 44 & 58,7 & 19 & 34,5 & 36 & 65,5 & 12 & 60,0 & 8 & 40,0 \\
\hline Cansaço & 65 & 86,7 & 10 & 13,3 & 48 & 87,3 & 7 & 12,7 & 17 & 85,0 & 3 & 15,0 \\
\hline Cognição & 51 & 68,0 & 24 & 32,0 & 34 & 61,8 & 21 & 38,2 & 17 & 85,0 & 3 & 15,0 \\
\hline Concentração & 49 & 65,3 & 26 & 34,7 & 32 & 58,2 & 23 & 41,8 & 17 & 85,0 & 3 & 15,0 \\
\hline Depressão & 38 & 50,7 & 37 & 49,3 & 25 & 45,5 & 30 & 54,5 & 13 & 65,0 & 7 & 35,0 \\
\hline Desamparo & 25 & 33,3 & 50 & 66,7 & 14 & 25,5 & 41 & 74,5 & 11 & 55,0 & 9 & 45,0 \\
\hline Desespero & 27 & 36,0 & 48 & 64,0 & 16 & 29,1 & 39 & 70,9 & 11 & 55,0 & 9 & 45,0 \\
\hline Insónia & 58 & 77,3 & 17 & 22,7 & 43 & 78,2 & 12 & 21,8 & 15 & 75,0 & 5 & 25,0 \\
\hline Medo & 41 & 54,7 & 34 & 45,3 & 32 & 58,2 & 23 & 41,8 & 9 & 45,0 & 11 & 55,0 \\
\hline Memória & 68 & 90,7 & 7 & 9,3 & 51 & 92,7 & 4 & 7,3 & 17 & 85,0 & 3 & 15,0 \\
\hline Nervosismo & 59 & 78,7 & 16 & 21,3 & 43 & 78,2 & 12 & 21,8 & 16 & 80,0 & 4 & 20,0 \\
\hline Orientação & 35 & 46,7 & 40 & 53,3 & 24 & 43,6 & 31 & 56,4 & 11 & 55,0 & 9 & 45,0 \\
\hline Pensamento & 52 & 69,3 & 23 & 30,7 & 34 & 61,8 & 21 & 38,2 & 18 & 90,0 & 2 & 10,0 \\
\hline Perceção & 42 & 56,0 & 33 & 44,0 & 27 & 49,1 & 28 & 50,9 & 15 & 75,0 & 5 & 25,0 \\
\hline Pesadelo & 40 & 53,3 & 35 & 46,7 & 31 & 56,4 & 24 & 43,6 & 9 & 45,0 & 11 & 55,0 \\
\hline Solidão & 49 & 65,3 & 26 & 34,7 & 35 & 63,6 & 20 & 36,4 & 14 & 70,0 & 6 & 30,0 \\
\hline Stress & 29 & 38,7 & 46 & 61,3 & 21 & 38,2 & 34 & 61,8 & 8 & 40,0 & 12 & 60,0 \\
\hline Tristeza & 48 & 64,0 & 27 & 36,0 & 33 & 60,0 & 22 & 40,0 & 15 & 75,0 & 5 & 25,0 \\
\hline Vontade de viver & 24 & 32,0 & 51 & 68,0 & 16 & 29,1 & 39 & 70,9 & 8 & 40,0 & 12 & 60,0 \\
\hline
\end{tabular}

Distribuição dos focos de Enfermagem pelos diagnósticos psiquiátricos

No sentido de conhecer a distribuição dos focos de Enfermagem em relação aos diagnósticos médicos, procedeu-seaumaanálise de distribuição de frequências (Tabela 2). Os resultados permitiram verificar que a grande parte dos focos de Enfermagem apareciam com maior frequência nos diagnósticos de depressão e demência, que foram os mais prevalentes. A análise permitiu também apurar que os focos relacionados com alterações do humor (tristeza, vontade de viver, autoestima, desolação, depressão, euforia, ideação suicida e tentativa de suicídio) e com perturbações ansiosas (ansiedade, stress, medo, nervosismo, 
cansaço, pesadelo, angústia, coping e desespero) apareciam com maior frequência nas pessoas com diagnóstico médico de depressão, ao passo que os focos relacionados com alterações do comportamento (agitação, comportamento agressivo, comportamento desorganizado e insónia) e da cognição (pensamento, delírio, crença errónea, alucinação, orientaçãa, atenção, concentração, cognição, aprendizagem, comunicação e confusão) surgiam mais nas pessoas com diagnóstico de demência.

Tabela 2

Distribuição de frequência dos focos de Enfermagem pelos diagnósticos médicos

\begin{tabular}{|c|c|c|c|c|c|c|c|c|}
\hline $\begin{array}{l}\text { Focos de } \\
\text { Enfermagem }\end{array}$ & $\begin{array}{l}\text { Perturb. } \\
\text { depressiva } \\
(n=27)\end{array}$ & $\begin{array}{c}\text { Demência } \\
(n=22)\end{array}$ & $\begin{array}{l}\text { Perturb. } \\
\text { bipolar } \\
(n=9)\end{array}$ & $\begin{array}{l}\text { Esquizofrenia } \\
\text { out. psicoses } \\
\quad(n=4)\end{array}$ & $\begin{array}{l}\text { Depend. } \\
\text { álcool } \\
(n=4)\end{array}$ & $\begin{array}{l}\text { Perturb. } \\
\text { ajustamento } \\
(n=4)\end{array}$ & $\begin{array}{l}\text { Perturb. } \\
\text { ansiedade } \\
(n=3)\end{array}$ & $\begin{array}{c}\text { Sem } \\
\text { diagnóstico } \\
(n=2)\end{array}$ \\
\hline Abuso de álcool & --- & 1 & --- & --- & 3 & 1 & --- & -- \\
\hline Agitação & 2 & 1 & --- & --- & --- & --- & --- & --- \\
\hline Alucinação & 1 & 6 & 2 & --- & --- & --- & --- & --- \\
\hline Angústia & 3 & 1 & --- & --- & --- & --- & --- & --- \\
\hline Ansiedade & 26 & 17 & 8 & 3 & 3 & 4 & 2 & --- \\
\hline Aprendizagem & 3 & 13 & --- & 2 & 1 & 1 & --- & --- \\
\hline Atenção & 17 & 20 & 6 & 4 & 4 & 2 & 1 & 2 \\
\hline Autocontrolo & 1 & 3 & --- & 1 & 3 & 1 & --- & --- \\
\hline Auto-estima & 13 & 11 & 3 & 1 & --- & 2 & 1 & -- \\
\hline Cansaço & 26 & 19 & 6 & 4 & 4 & 3 & 3 & --- \\
\hline Cognição & 17 & 19 & 3 & 3 & 4 & 2 & 1 & 2 \\
\hline Comp. Agressivo & --- & 6 & 1 & --- & --- & --- & --- & --- \\
\hline Comp. Desorganizado & 1 & 2 & -- & 1 & --- & --- & -- & --- \\
\hline Comunicação & 7 & 9 & --- & 1 & 3 & --- & 1 & 1 \\
\hline Concentração & 15 & 18 & 6 & 2 & 4 & 2 & 1 & 1 \\
\hline Confusão & 2 & 13 & --- & 2 & 1 & 1 & -- & --- \\
\hline Coping & 8 & 4 & 3 & 1 & 1 & 2 & 1 & --- \\
\hline Crença errónea & --- & 4 & 2 & --- & 1 & --- & --- & --- \\
\hline Delírio & --- & 4 & 2 & --- & 1 & --- & --- & --- \\
\hline Depressão & 19 & 11 & 1 & 2 & 2 & 2 & 1 & --- \\
\hline Desamparo & 10 & 9 & 1 & --- & 3 & 2 & --- & --- \\
\hline Desespero & 11 & 9 & 2 & 1 & 1 & 3 & --- & --- \\
\hline Desolação & 11 & 3 & --- & --- & --- & 1 & --- & --- \\
\hline Euforia & --- & 1 & 2 & --- & --- & --- & --- & --- \\
\hline Falta de esperança & 9 & 2 & 1 & --- & 2 & 1 & 1 & --- \\
\hline Força de vontade & 8 & 5 & --- & --- & 1 & 1 & 1 & --- \\
\hline Ideação suicida & 7 & 3 & 3 & 1 & --- & 3 & 1 & --- \\
\hline Insónia & 25 & 16 & 7 & 2 & 4 & 2 & 2 & --- \\
\hline Medo & 19 & 7 & 6 & 1 & 3 & 4 & 1 & --- \\
\hline Memória & 25 & 22 & 7 & 3 & 4 & 2 & 3 & 2 \\
\hline Nervosismo & 19 & 17 & 9 & 4 & 3 & 4 & 3 & --- \\
\hline Orientação & 8 & 17 & 1 & 2 & 4 & 1 & 1 & 1 \\
\hline Pensamento & 17 & 19 & 4 & 3 & 4 & 2 & 1 & 2 \\
\hline Perceção & 9 & 17 & 6 & 2 & 4 & 2 & 1 & 1 \\
\hline Pesadelo & 14 & 12 & 4 & 2 & 4 & 4 & --- & --- \\
\hline Satisfação conjugal & 5 & 3 & 1 & --- & 1 & 1 & --- & --- \\
\hline Socialização & 4 & 9 & 2 & 1 & 3 & 1 & --- & --- \\
\hline Solidão & 19 & 16 & 5 & 2 & 4 & 2 & 1 & --- \\
\hline Stress & 17 & 4 & 2 & 1 & 2 & 2 & 1 & --- \\
\hline Tentativa de suicídio & 4 & --- & --- & 1 & --- & 1 & --- & 1 \\
\hline Tristeza & 23 & 13 & 2 & 3 & 2 & 3 & 2 & --- \\
\hline Vontade de viver & 11 & 5 & 3 & 1 & 1 & 3 & --- & --- \\
\hline
\end{tabular}


Análise da relação entre os focos de Enfermagem e as variáveis sociodemográficas, clínicas e de funcionalidade

Relativamente à associação entre os focos de Enfermagem e os diagnósticos psiquiátricos, tendo em conta que os diagnósticos de depressão e demência eram os mais comuns, representando em conjunto 65,3\% da amostra, procurou-se analisar a existência de eventuais associações entre estes. Procedeu-se ainda a uma análise de associações e de correlações entre os focos de Enfermagem e alguns fatores sociodemográficos (idade e género), clínicos e de funcionalidade.

Os resultados revelaram existir uma associação positiva significativa entre o diagnóstico de demência e a idade $\left(r_{p h}=, 35 ; p=, 002\right)$, sugerindo que mais idade estava associada ao diagnóstico de demência, não se verificando o mesmo em relação à depressão. No que diz respeito ao género, o teste de Quiquadrado efetuado para o diagnóstico de demência não permitiu concluir de forma significativa que existisse diferença entre homens e mulheres $\left(\chi^{2}(1)\right.$ $=3,23 ; p=, 072)$. Relativamente ao diagnóstico de depressão, verificou-se uma diferença significativa entre géneros $\left(\chi^{2}(1)=5,22 ; p=, 022\right)$, concluindose que os homens $(15,0 \%)$ estão menos sujeitos a depressão do que as mulheres (43,6\%).

No que diz respeitoà relação dos focos de Enfermagem com as variáveis clínicas e sociodemográficas (Tabela 3), foram encontradas correlações positivas entre a idade e os focos aprendizagem, atenção, cognição, confusão, força de vontade, orientação e pensamento. Por outro lado, foram encontradas correlações negativas entre esta variável e os focos ansiedade, coping, desespero, desolação, ideação suicida, stress, tristeza e vontade de viver. No que diz respeito ao género, verificou-se apenas uma associação positiva entre o foco stress e esta variável $\left(\chi^{2}(1)=4,01 ; p\right.$ $=, 045)$, concluindo-se que as mulheres $(45,5 \%)$ apresentavam mais alterações neste foco do que os homens (20,0\%).

Relativamente à relação dos focos de Enfermagem com os diagnósticos psiquiátricos (depressão e demência), considerando as associações encontradas, procurou-se verificar a importância das variáveis idade e género nesta relação, isto é, pretendeu-se saber se a relação observada estava a ser influenciada por estas variáveis sociodemográficas. Os dados obtidos, depois de controlados para a idade e o género, permitiram verificar que apenas os focos alucinação $(p=, 011)$, confusão $(p<, 001)$, orientação $(p<$ ,035), aprendizagem $(p=, 001)$, comportamento agressivo $(p<, 001)$ e perceção $(p=, 044)$ surgiam correlacionados positivamente com a demência. Do mesmo modo, em relação à depressão, apenas se verificaram correlações positivas com os focos tristeza $(p=, 003)$, depressão $(p=, 016)$, desolação $(p=$ $, 002)$ e stress $(p=, 005)$. Por outro lado, os focos aprendizagem $(p=, 046)$, comportamento agressivo $(p=, 035)$, confusão $(p=, 017)$ e perceção $(p=$ ,004), apareciam correlacionados negativamente com o diagnóstico médico de depressão. Relativamente aos restantes focos de Enfermagem não se verificou a existência de correlação com os diagnósticos médicos apresentados.

\section{Tabela 3}

Frequências, associações e correlações entre os focos de Enfermagem e os diagnósticos demência e depressão, a idade e o género $(n=75)$

\begin{tabular}{lccccccccc}
\hline \multirow{2}{*}{ Focos de Enfermagem } & \multicolumn{3}{c}{ Demência } & \multicolumn{2}{c}{ Depressão } & \multicolumn{3}{c}{ Idade } & \multicolumn{3}{c}{ Género $(n=20) /(n=55)$} \\
\cline { 2 - 9 } & $\%$ & $p$ & $\%$ & $p$ & $\left(r_{p h}\right)$ & $P$ & $\mathrm{M}(\%)$ & $\mathrm{F}(\%)$ & $p$ \\
\hline Alucinação & 27,3 &, 009 & 3,7 &, 097 &, 12 &, 304 & 10,0 & 12,7 &, 748 \\
Ansiedade & 77,3 &, 306 & 96,3 &, 029 &,- 31 &, 006 & 75,0 & 87,3 &, 200 \\
Aprendizagem & 50,1 &, 001 & 11,1 &, 022 &, 41 &, 001 & 35,0 & 23,6 &, 325 \\
Atenção & 90,9 &, 037 & 63,0 &, 080 &, 30 &, 008 & 70,0 & 76,3 &, 575 \\
Cognição & 86,4 &, 028 & 63,0 &, 483 &, 32 &, 005 & 70,0 & 67,3 &, 823 \\
Comp. Agressivo & 27,3 &, 001 & 0,0 &, 037 &,- 00 &, 993 & 10,0 & 9,1 &, 905 \\
Confusão & 59,1 &, 001 & 7,4 &, 007 &, 39 &, 001 & 35,0 & 21,8 &, 246 \\
Coping & 18,2 &, 284 & 27,6 &, 663 &,- 25 &, 028 & 20,0 & 29,1 &, 431 \\
Crença errónea & 18,2 &, 090 & 0,0 &, 037 &, 08 &, 518 & 15,0 & 7,3 &, 309 \\
Delírio & 18,2 &, 090 & 0,0 &, 037 &, 08 &, 518 & 15,0 & 7,3 &, 309 \\
Depressão & 5,0 &, 941 & 70,4 &, 010 &,- 19 &, 111 & 45,0 & 52,7 &, 554
\end{tabular}




\begin{tabular}{llllllllll} 
Desespero & 40,9 &, 568 & 40,7 &, 521 &,- 23 &, 047 & 30,0 & 38,2 &, 514 \\
Desolação & 13,6 &, 375 & 40,7 &, 001 &,- 26 &, 026 & 10,0 & 23,6 &, 192 \\
Força de vontade & 22,7 &, 849 & 29,6 &, 188 &, 31 &, 007 & 30,0 & 18,2 &, 269 \\
Ideação suicida & 13,6 &, 176 & 25,9 &, 770 &,- 26 &, 027 & 35,0 & 20,0 &, 179 \\
Insónia & 72,7 &, 539 & 92,6 &, 018 &,- 19 &, 106 & 65,0 & 81,8 &, 124 \\
Medo & 31,8 &, 010 & 70,4 &, 040 &,- 23 &, 050 & 40,0 & 60,0 &, 124 \\
Orientação & 77,3 &, 001 & 29,6 &, 027 &, 54 &, 001 & 60,0 & 41,8 &, 163 \\
Pensamento & 86,4 &, 039 & 63,0 &, 370 &, 30 &, 008 & 70,7 & 69,1 &, 940 \\
Perceção & 77,3 &, 017 & 33,3 &, 003 &, 16 &, 170 & 60,0 & 54,5 &, 674 \\
Stress & 18,2 &, 019 & 63,0 &, 001 &,- 28 &, 015 & 20,0 & 45,5 &, 045 \\
Tristeza & 59,1 &, 568 & 85,2 &, 004 &,- 26 &, 026 & 65,0 & 63,6 &, 913 \\
Vontade de viver & 22,7 &, 267 & 40,7 &, 224 &,- 25 &, 031 & 35,0 & 30,9 &, 737 \\
\hline
\end{tabular}

Relativamente à associação entre os focos de Enfermagem e alguns fatores clínicos e de funcionalidade, efetuaram-se análises de correlação para os focos mais comuns, tendo por base as pontuações globais obtidas nas escalas usadas no protocolo de avaliação. Os resultados revelaram existir correlação (positiva e negativa) entre vários focos de Enfermagem e os resultados de alguns destes instrumentos (Tabela 4). Verificou-se que os focos do domínio cognitivo apareciam associados a pontuações mais baixas nos testes cognitivos (MMSE/CDT) e a menor funcionalidade (BI/LI) dos sujeitos. Estes dados sugeriam que as pessoas com mais dificuldades cognitivas apresentariam maiores necessidades de
Enfermagem em alguns destes domínios, o que, por sua vez, também impõe dificuldades na satisfação das suas necessidades básicas e sobretudo, das instrumentais. Por outro lado, os focos relacionados com o humor e a ansiedade apareciam associados a pontuações mais elevadas nas respetivas escalas (GDS/ZAS), o que permitiu concluir que a presença de níveis elevados de ansiedade e depressão, nos sujeitos, determinaria maiores necessidades de Enfermagem, em diferentes focos destes domínios psicológicos. Por sua vez, os problemas ao nível dos focos do domínio do humor e da ansiedade não comprometiam tanto a funcionalidade e a realização das AVD.

Tabela 4

Correlações entre os focos de Enfermagem e as pontuações globais dos instrumentos de avaliação clínica $(n=75)$

\begin{tabular}{lccccccccccccc}
\hline \multirow{2}{*}{ Focos de Enfermagem } & \multicolumn{1}{c}{ MMSE } & \multicolumn{1}{c}{ CDT } & \multicolumn{3}{c}{ GDS } & \multicolumn{3}{c}{ ZAS } & \multicolumn{3}{c}{ IB } & \multicolumn{3}{c}{ IL } \\
\cline { 2 - 12 } & $\left(r_{p h}\right)$ & $p$ & $\left(r_{p h}\right)$ & $p$ & $\left(r_{p h}\right)$ & $p$ & $\left(r_{p h}\right)$ & $p$ & $\left(r_{p h}\right)$ & $p$ & $\left(r_{p h}\right)$ & $p$ \\
\hline Ansiedade &, 16 &, 183 &,- 02 &, 876 &, 42 &, 000 &, 66 &, 000 &,- 08 &, 510 &,- 04 &, 726 \\
Aprendizagem &,- 80 &, 000 &,- 57 &, 000 &, 14 &, 234 &,- 03 &, 810 &,- 57 &, 000 &,- 70 &, 000 \\
Atenção &,- 57 &, 000 &,- 71 &, 000 &, 13 &, 256 &, 02 &, 840 &,- 35 &, 002 &,- 51 &, 000 \\
Cansaço &,- 03 &, 786 &,- 16 &, 178 &, 44 &, 000 &, 43 &, 000 &,- 08 &, 475 &,- 14 &, 226 \\
Cognição &,- 53 &, 000 &,- 65 &, 000 &, 26 &, 027 &, 20 &, 088 &,- 33 &, 004 &,- 45 &, 000 \\
Concentração &,- 47 &, 000 &,- 70 &, 000 &, 23 &, 045 &, 17 &, 141 &,- 31 &, 007 &,- 47 &, 000 \\
Depressão &,- 03 &, 786 &,- 19 &, 105 &, 81 &, 000 &, 46 &, 000 &,- 14 &, 217 &,- 14 &, 234 \\
Insónia &, 20 &, 081 &,- 03 &, 813 &, 12 &, 325 &, 36 &, 001 &, 05 &, 664 &, 08 &, 497 \\
Medo &, 24 &, 041 &, 11 &, 355 &, 29 &, 011 &, 42 &, 000 &, 09 &, 428 &, 07 &, 548 \\
Memória &,- 30 &, 009 &,- 32 &, 005 &, 11 &, 364 &, 10 &, 392 &,- 21 &, 077 &,- 28 &, 014 \\
Orientação &,- 73 &, 000 &,- 59 &, 000 &, 10 &, 380 &,- 14 &, 225 &,- 45 &, 000 &,- 56 &, 000 \\
Pensamento &,- 51 &, 000 &,- 68 &, 000 &, 23 &, 047 &, 17 &, 153 &,- 32 &, 006 &,- 48 &, 000 \\
Peceção &,- 41 &, 000 &,- 63 &, 000 &, 18 &, 125 &, 19 &, 115 &,- 22 &, 050 &,- 44 &, 000 \\
Pesadelo &,- 05 &, 664 &,- 16 &, 175 &, 21 &, 070 &, 37 &, 001 &,- 06 &, 627 &,- 07 &, 540 \\
Solidão &,- 27 &, 019 &,- 37 &, 001 &, 64 &, 000 &, 41 &, 000 &,- 21 &, 070 &,- 34 &, 003 \\
Tristeza &,- 05 &, 643 &,- 02 &, 854 &, 69 &, 000 &, 43 &, 000 &,- 02 &, 848 &, 04 &, 719 \\
\hline
\end{tabular}




\section{Discussão}

Os resultados apresentados colocam a depressão e a demência como os diagnósticos médicos mais frequentes, o que está em conformidade com os dados de outros autores (Apóstolo et al., 2011; Benedetti et al., 2008; Canabrava et al., 2012; Passos et al., 2010, 2012). Estes dados estão também em linha com os resultados dos instrumentos aplicados, uma vez que a maioria dos participantes apresentava défice cognitivo no MMSE e no CDT e depressão na GDS.

Associado a estes diagnósticos médicos encontrouse um vasto conjunto de alterações psicológicas, cognitivas e comportamentais, que constituem focos de atenção relevantes para a prática de Enfermagem. Grande parte destes focos de Enfermagem relacionados com a saúde mental, na sua maioria do domínio cognitivo e das emoções, parecem ser comuns nas pessoas mais velhas, tendo em conta os resultados apresentados neste estudo e noutras pesquisas, já realizadas (Passos et al., 2010, 2012), bem como nos dados de outros autores (Sequeira, 2010).

Por outro lado, verificou-se que os focos de Enfermagem do domínio cognitivo apareciam correlacionados com maior idade das pessoas, contribuindo para a existência de vários diagnósticos de Enfermagem como é o caso da atenção diminuída, cognição comprometida, confusão atual, força de vontade diminuída, orientação comprometida e pensamento anormal, o que também tem sido referido por outros autores (Ferreira, Tavares, \& Rodrigues, 2011; Santos, et al., 2008; Teixeira \& Fernandes, 2003). Verificou-se também que os sujeitos com menos idade apresentavam mais alterações ao nível dos focos relacionados com o domínio psicológico (ansiedade e humor), originando a presença de diagnósticos como ansiedade atual, coping comprometido, desespero atual, desolação atual, stress elevado, ideação suicida presente, tristeza presente e vontade de viver diminuída. Outros autores têm também identificado a presença de alterações em alguns destes domínios nas pessoas mais velhas, contudo não fazem distinção relativamente à idade (Santos et al., 2008; Teixeira \& Fernandes, 2003). A presença de muitos destes focos, sobretudo os do domínio cognitivo, cria várias dificuldades aos idosos e compromete a realização dos seus objetivos de vida. Esta condição, apesar de exigir uma atenção particular, não tem tido a melhor resposta no contexto dos cuidados de Enfermagem, verificando-se que as prioridades têm sido mais orientadas no sentido da satisfação das necessidades básicas em detrimento da estimulação cognitiva e da promoção da autonomia destas pessoas. Do mesmo modo, em contextos de psiquiatria e saúde mental, o sofrimento psicológico e outras alterações emocionais, não estão a obter a melhor resposta da parte dos enfermeiros, dada a diminuição de recursos que se tem verificado e a excessiva focalização nos aspetos anteriormente referidos.

Por outro lado, verificou-se que os focos do domínio cognitivo e comportamental, aparecem mais associados ao diagnóstico de demência, enquanto que os do domínio psicológico, relacionados com as emoções, aparecem mais associados ao diagnóstico médico de depressão, dados que surgem em conformidade com estudos anteriores (Passos et al., 2010, 2012). Outros autores têm também referido o predomínio dos problemas cognitivos e comportamentais associados à demência (Sequeira 2010), do mesmo modo que alguns estudos relacionam as alterações ao nível das emoções com o diagnóstico de depressão (Unutzer, 2007). Também se verificou que, depois de controlados os fatores sociodemográficos, apenas se mantinha correlação positiva entre os focos alucinação, aprendizagem, comportamento agressivo, confusão, orientação e perceção e o diagnóstico médico de demência. Por outro lado, apareciam correlacionados positivamente com o diagnóstico médico de depressão, apenas os focos depressão, desolação, stress e tristeza. Tendo em conta estas correlações, os resultados permitem concluir que estes focos podem ser determinantes importantes destes diagnósticos médicos.

Os resultados apontaram também para a existência de correlação significativa entre os focos de Enfermagem do domínio cognitivo e as pontuações obtidas nos testes cognitivos, bem como em relação aos índices de funcionalidade, verificando-se que estas alterações tinham um grande impacto nas AVD, o que está em linha com a perspetiva de outros autores (Araújo et al., 2007, 2008; Ferreira et al., 2011). No entanto, estas dificuldades predominavam ao nível das atividades instrumentais, como tem sido também verificado noutros estudos (Apóstolo et al., 2011; Sequeira, 2010).

Por outro lado, os focos do domínio psicológico (humor e ansiedade), que apresentavam frequências mais elevadas, apareciam correlacionados com 
pontuações mais altas nas escalas de depressão e ansiedade (GDS/ZAS), sugerindo que as pessoas com níveis mais elevados de ansiedade e depressão apresentavam mais problemas ao nível das emoções, o que está em linha com os dados da literatura (Unutzer, 2007). No entanto, as alterações em alguns focos do domínio cognitivo, como é o caso da cognição, concentração e pensamento, apareciam também correlacionadas com níveis mais elevados de depressão, que é corroborado por estudos prévios (Apóstolo et al., 2011; Gonçalves \& Martín, 2007). Estes dados sugerem a existência de comorbilidades em termos de sintomatologia cognitiva e emocional que provoca sofrimento e grande disfuncionalidade nestas pessoas. Esta evidência realça a importância de uma boa avaliação, de modo a identificar com rigor a natureza dos problemas, as suas causas e as necessidades associadas.

Neste sentido, torna-se importante desenvolver estratégias e métodos de avaliação eficazes, que permitam identificar com rigor os verdadeiros problemas e necessidades das pessoas mais velhas e os seus determinantes. $\mathrm{O}$ estudo pormenorizado destes eventos é fundamental para a valorização dos fatores associados a estas necessidades e para o planeamento de uma resposta assistencial em conformidade com a natureza clínica e social dos fenómenos que caraterizam o envelhecimento e as problemáticas destes utentes.

Os dados obtidos parecem-nos sensíveis à natureza clínica dos diagnósticos médicos mais comuns (depressão e demência). Apesar dos utentes estarem a beneficiar de tratamento/intervenção, quer médico quer de Enfermagem, verificou-se a presença de um vasto conjunto de sinais e sintomas, identificados através dos instrumentos utilizados, que muitas vezes dominam o quadro clínico, e cujo reconhecimento constitui um desafio e um alvo prioritário de atenção da prática de Enfermagem.

Apesar do rigor utilizado na metodologia e na análise dos dados obtidos através do presente estudo, persistem algumas limitações, uma vez que houve dificuldade em identificar a presença ou ausência de alguns focos de Enfermagem, devido à escassa informação na descrição do respetivo conceito, bem como a semelhança entre o seu conteúdo. Isto devese ao facto de a CIPE, em alguns focos, apresentar conceitos com caraterísticas sobreponíveis, o que dificulta a valorização da informação colhida, concretamente, a identificação rigorosa do foco em causa e das respetivas alterações.

\section{Conclusão}

O presente estudo permitiu identificar e hierarquizar a prevalência dos focos de Enfermagem de saúde mental em pessoas mais velhas, bem como conhecer a sua relação com algumas variáveis sociodemográficas, clínicas e de funcionalidade, contribuiu para uma melhor perceção dos problemas que afetam esta população. Deste modo, esta investigação possibilitou retirar implicações na realização das atividades de vida diária, bem como no bem-estar físico e psicológico destes idosos.

A demência e a depressão constituem determinantes importantes de focos e diagnósticos de Enfermagem relacionados com a saúde mental das pessoas mais velhas, evidenciando a importância de uma avaliação rigorosa das diferentes dimensões e eventos que caracterizam o envelhecimento humano e o processo de adaptação destas pessoas às alterações físicas e psicológicas e aos contextos de vida.

Este trabalho permitiu assim, perspetivar os próximos passos, concretamente no que diz respeito à elaboração de um Catálogo CIPE dirigido às necessidades do idoso em saúde mental e psiquiatria e ao desenvolvimento de um questionário de avaliação clínica de enfermagem, neste âmbito.

\section{Referências bibliográficas}

Apóstolo, J. L. A., Cardoso, D. F. B., Marta, L. M. G., \& Amaral, T. I. O. (2011). Efeito da estimulação cognitiva em idosos. Revista de Enfermagem Referência, 3(5), 193-201. doi:10.12707/ RIII11104

Araújo, F., Ribeiro, J. L. P., Oliveira, A., \& Pinto, C. (2007). Validação do Índice de Barthel numa amostra de idosos não institucionalizados. Revista Portuguesa de Saúde Pública, 25(2), 59-66

Araújo, F., Ribeiro, J. L. P., Oliveira, A., Pinto, C., \& Martins, T. (2008). Validação da escala de Lawton e Brody numa amostra de idosos não institucionalizados. In Actas do $7^{\circ}$ Congresso Nacional de Psicologia da Saúde (pp. 655-659). Portugal: Universidade do Porto.

Barreto, J., Leuschner, A., Santos, F., \& Sobral, M. (2003). Escala de depressão geriátrica. Lisboa, Portugal: Grupo Estudos de Envelhecimento Cerebral e Demências. 
Benedetti, T. R. B., Borges, L. J., Petroski, E. L., \& Gonçalves, L. H. T. (2008). Actividade física e estado de saúde mental de idosos. Revista Saúde Pública, 42 (2), 302-307. doi:10.1590/ S0034-89102008005000007

Cacho, J., García-García, R., Arcaya, J., Vicente, J. L., \& Lantada, N. (1999). Una propuesta de aplicación y pontuación del test del reloj en la enfermedad de Alzheimer. Revista Neurologia, 28(7), 648-655.

Canabrava, D. S., Brusamarello, T., Capistrano, F. C., Mazza, V. A., Mercês, N. N. A., \& Maftum, M. A. (2012). Diagnóstico e intervenções à pessoa com transtorno mental com base na consulta de enfermagem. Cogitare Enfermagem, 17(4), 661-668.

Conselho Internacional de Enfermeiros. (2011). CIPE versão 2: Classificação internacional para a prática de enfermagem. Lisboa, Portugal: Ordem dos Enfermeiros.

Fernandes, L. (2004). Factores biopsicológicos e dinâmica familiar na doença asmática (Tese de Doutoramento não publicada). Universidade do Porto, Faculdade de Medicina, Portugal.

Ferreira, P. C. S., Tavares, D. M. S., \& Rodrigues, R. A. P. (2011). Características sociodemográficas, capacidade funcional e morbidades entre idosos com e sem declínio cognitivo. Acta Paulista de Enfermagem, 24(1), 29-35.

Gonçalves, D. C., \& Martín, I. (2007). Intervenção na depressão geriátrica através da reminiscência. Psicologia Argumento, 25(51), 371-384

Morgado, J., Rocha, C. S., Maruta, C., Guerreiro, M., \& Martins I. P. (2009). New normative values of Mini-Mental State Examination. Sinapse, 9(2), 10-16.
Passos, J., Sequeira, C., \& Fernandes, L. (2010). Implicações da saúde mental no envelhecimento activo: Uma perspectiva de enfermagem. In C. Sequeira \& L. Sá (Coords.), Do diagnóstico à intervenção em saúde mental. Porto, Portugal: Sociedade Portuguesa de Enfermagem de Saúde Mental.

Passos, J., Sequeira, C., \& Fernandes, L. (2012). Prevalência dos focos de enfermagem de saúde mental em pessoas mais velhas: Resultados da pesquisa documental realizada num serviço de psiquiatria. Revista Portuguesa de Enfermagem de Saúde Mental, 7, 7-12.

Santos, A. S. R., Souza, P. A., Valle, A. M. D., Cavalcanti, A. C. D., Sá, S. P. C., \& Santana, R. F. (2008). Caracterização dos diagnósticos de enfermagem identificados em prontuários de idosos: Um estudo retrospectivo. Texto Contexto Enfermagem, 17(1), 141-149. doi:10.1590/S0104-07072008000100016

Sequeira, C. (2010). Cuidar de idosos com dependência física e mental. Lisboa, Portugal: Lidel.

Serra, A. V., Ponciano, E., \& Relvas, J. (1982). Aferição da escala de auto-avaliação de ansiedade, de Zung, numa amostra de população portuguesa - II.: - Sua avaliação como instrumento de medida. Psiquiatria Clínica, 3(4), 203-213.

Teixeira, M. B., \& Fernandes, R. A. Q. (2003). Diagnósticos de enfermagem identificados em idosos com distúrbio mental. Revista Brasileira de Enfermagem, 56(6), 619-623. doi:10.1590/\$0034-71672003000600005

Unutzer, J. (2007). Clinical practice. Late-life depression. The New England Journal of Medicine, 357(22), 2269-2276. doi:10.1056/NEJMcp073754

World Health Organization. (1978). International classification of diseases (ICD-9). Geneva, Switzerland: Autor 
